\title{
Tanshinone IIA decreases the migratory ability of AGS cells by decreasing the protein expression of matrix metalloproteinases, nuclear factor $\mathbf{\kappa B}$-p65 and cyclooxygenase-2
}

\author{
CHIN-CHENG SU ${ }^{1-4}$ \\ ${ }^{1}$ Tumor Research Center of Integrative Medicine; ${ }^{2}$ Comprehensive Breast Cancer Center; ${ }^{3}$ Department of Surgery, \\ Changhua Christian Hospital, Changhua, Changhua 50006; ${ }^{4}$ School of Chinese Medicine, \\ College of Chinese Medicine, China Medical University, Taichung 40402, Taiwan, R.O.C.
}

Received February 7, 2015; Accepted October 22, 2015

DOI: $10.3892 / \mathrm{mmr} .2015 .4658$

\begin{abstract}
During progression of gastric cancer, degradation of the extracellular matrix by matrix metalloproteinases (MMPs) has been associated with poor prognosis Tanshinone IIA (Tan-IIA) exerts antitumor activity in a variety of human cancer cells. It is extracted from Danshen (Salviae miltiorrhizae radix), and induces apoptosis and inhibits the proliferation of gastric cancer cells. However, the molecular mechanisms underlying the inhibition of migration in gastric cancer by Tan-IIA have not been fully elucidated. In the present study, AGS cell migration ability was evaluated using a wound-healing assay. The protein expression levels of nuclear factor (NF)- $\mathrm{B}-\mathrm{p} 65$, cyclooxygenase (COX)-2, MMP-2, -7 , and -9 and $\beta$-actin in AGS cells were measured by western blotting. The results demonstrated that AGS cells treated with Tan-IIA exhibit decreased protein expression levels of NF-кB-p65, COX-2, and MMP-2, -7 and -9. The results also indicate that Tan-IIA inhibits migration ability in a dose- and time-dependent manner. These findings demonstrate that Tan-IIA inhibits the migration ability of AGS human gastric cancer cells and that decreasing the protein expression of NF- $\mathrm{NB}-\mathrm{p} 65, \mathrm{COX}-2$, and MMP-2, -7 and -9 may be an underlying molecular mechanism.
\end{abstract}

\section{Introduction}

Tanshinone IIA (Tan-IIA; $\mathrm{C}_{19} \mathrm{H}_{18} \mathrm{O}_{3}$ ) is extracted from Danshen (Salviae miltiorrhizae radix) $(1,2)$. Tan-IIA exerts antitumor activity in a variety of human cancer cells, such as

Correspondence to: Dr Chin-Cheng Su, Tumor Research Center of Integrative Medicine, Changhua Christian Hospital, 135 Nan-Hsiao Street, Changhua, Changhua 50006, Taiwan, R.O.C.

E-mail: succ.maeva@msa.hinet.net

Key words: tanshinone IIA, AGS gastric carcinoma cells, migration, matrix metalloproteinases, nuclear factor $\kappa \mathrm{B}-\mathrm{p} 65$ lung (3), breast (4,5), hepatic (6), pancreatic (7) and colon (8). These studies (3-8) suggest that Tan-IIA may be administered as a complementary therapeutic agent to treat gastric cancer. Furthermore, our previous studies demonstrated that Tan-IIA inhibits the proliferation of AGS cells in a time- and dose-dependent manner, by decreasing the level of protein expression of binding immunoglobulin (Ig) protein, myeloid cell leukemia 1 protein, B-cell lymphoma-extra-large and translationally-controlled tumor protein, and increasing caspase-12, C/EBP-homologous protein, Bcl-2-associated X protein, and caspase-12, -9 and -3 to induce apoptosis (9). The half-maximal inhibitory concentration was 5.5, 3.7 and $3.5 \mu \mathrm{g} / \mathrm{ml}$ at 24,48 and $72 \mathrm{~h}$, respectively (9). In addition, Tan-IIA was demonstrated to inhibit AGS human gastric cancer cells by increasing the protein expression level of phosphorylated (p)-p38 and p-Jun-amino-terminal kinase, and decreasing that of $\mathrm{p}$-extracellular-signal-regulated kinases to induce $\mathrm{G}_{2} / \mathrm{M}$ phase arrest. In addition, Tan-IIA increased the protein expression levels of tumor necrosis factor- $\alpha$, FAS ligand, and caspase- 8 and -3 to induce apoptosis (10). It is well documented that nuclear factor $(\mathrm{NF})-\kappa \mathrm{B}$ functions as a tumor promoter in inflammation-associated cancer and is, therefore, a potential target for cancer prevention in chronic inflammatory diseases (11). During malignant tumor metastasis, the degradation of the extracellular matrix (ECM) is mediated by matrix metalloproteinases (MMPs) (12-14). As the underlying mechanisms of Tan-IIA inhibiting the migration ability of gastric cancer cells remain to be elucidated, this was the aim of the present study, as well as evaluating the levels of metastatic-associated protein expression in AGS human gastric cancer cells.

\section{Materials and methods}

Materials. The AGS human gastric adenocarcinoma cell line [Bioresource Collection and Research Center (BCRC); BCRC no. 60102] was obtained from the Food Industry Research and Development Institute (Hsinchu, Taiwan). Tan-IIA(CAS no. 568-72-9) was obtained from Sigma-Aldrich (St. Louis, MO, USA). Ham's F-12K (Kaign's) Medium, fetal bovine serum (FBS), 1\% penicillin/streptomycin and 


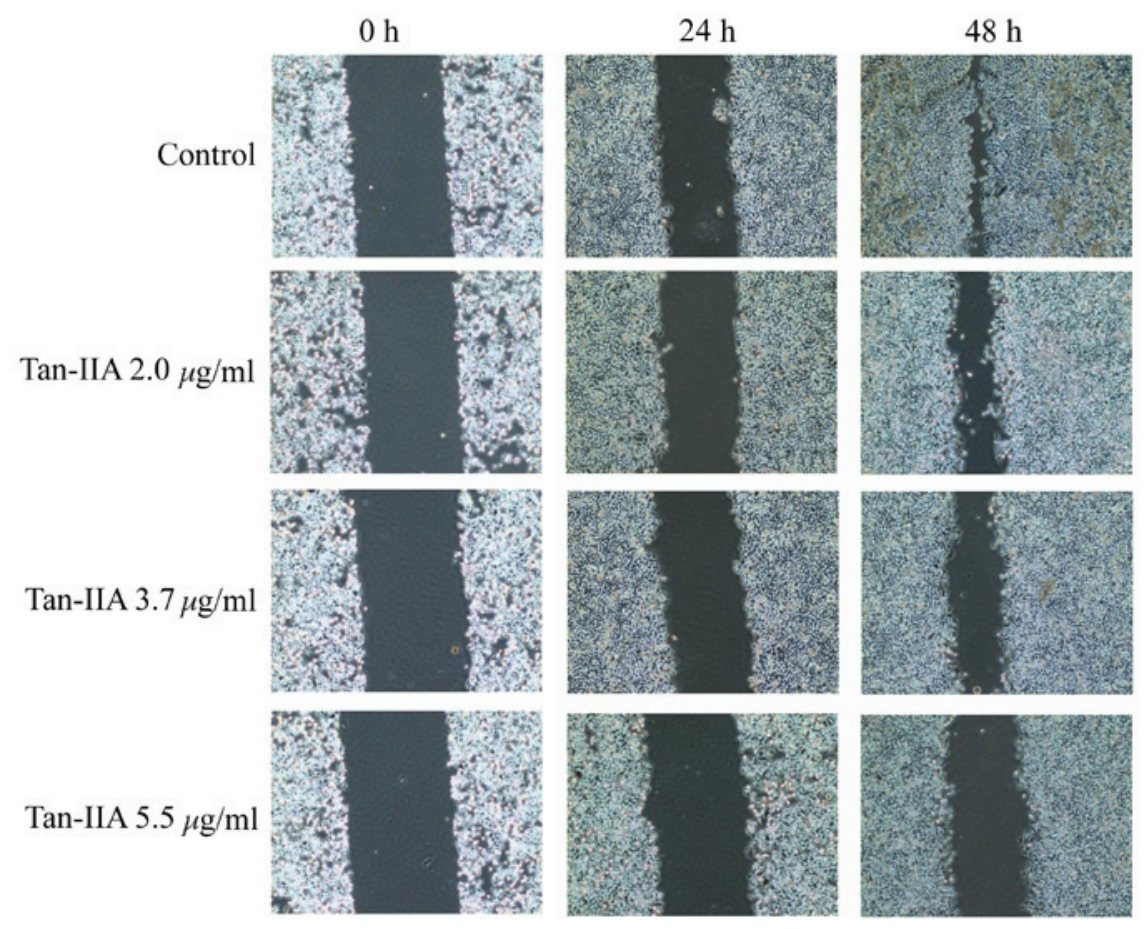

Figure 1. Wound-healing assays. AGS cells were treated with various concentrations of Tan-IIA (0, 2.0, $3.7 \mathrm{and} 5.5 \mu \mathrm{g} / \mathrm{ml})$ for $24 \mathrm{or} 48 \mathrm{~h}$, and the images were captured to compare the cell migration for wound closure. The results demonstrate that Tan-IIA inhibits the migration of AGS cells. Tan-IIA, Tanshinone IIA.

glutamine were obtained from Thermo Fisher Scientific, Inc. (Gibco; Waltham, MA, USA). The polyclonal rabbit anti-human NF-кB-p65 (cat. no. 6956; molecular weight, $65 \mathrm{kDa})$, monoclonal rabbit anti-human cyclooxygenase (COX)-2 (cat. no. 12282; molecular weight, $74 \mathrm{kDa}$ ) and monoclonal rabbit anti-human MMP-2 (cat. no. 13132; molecular weight, $72 \mathrm{kDa}$ ) antibodies were all obtained from Cell Signaling Technology, Inc. (Danvers, MA, USA). Polyclonal goat anti-human MMP-7 (cat. no. NB600-1069; molecular weight, $28 \mathrm{kDa}$ ) and polyclonal rabbit anti-human MMP-9 (cat. no. NBP1-57940; molecular weight, 78kDa) antibodies were obtained from Novus Biologicals, LLC (Littleton, CO, USA). Sodium deoxycholate, leupeptin, Triton $\mathrm{X}-100$, Tris-HCl, ribonuclease-A, sodium pyruvate, HEPES, dimethyl sulfoxide, Tween- 20 and mouse anti- $\beta$-actin antibodies were obtained from Sigma-Aldrich. BioMax film was obtained from Kodak (Rochester, NY, USA), and potassium phosphate and $0.2-\mathrm{mm}$ polyvinylidene difluoride (PVDF) membranes were purchased from Merck Millipore (Darmstadt, Germany).

Cell culture was conducted as described in a previous study (9). The AGS cells were placed into $75-\mathrm{cm}^{2}$ tissue culture flasks and maintained in Ham's F-12K Medium containing $10 \%$ heat-inactivated FBS, $100 \mathrm{U} / \mathrm{ml}$ penicillin and $100 \mu \mathrm{g} / \mathrm{ml}$ streptomycin. Cells were grown for $48-72 \mathrm{~h}$ at $37^{\circ} \mathrm{C}$ in a humidified atmosphere of $5 \% \mathrm{CO}_{2}$.

Wound-healing assays were performed as described in a previous study (15). Cells were plated at a density of $1 \times 10^{6}$ cells per $60-\mathrm{mm}$ Petri dish in complete medium for $16-20 \mathrm{~h}$. Different concentrations $(0,2.0,3.7$ and $5.5 \mu \mathrm{g} / \mathrm{ml})$ of Tan-IIA were administered for 0,24 , and $48 \mathrm{~h}$ and, once the cells reached confluency, a plastic pipette tip was drawn across the center of the plate to produce a clean, wide, wound area.
Cell movement into the wound area was examined under an Olympus 1X81 microscope (Olympus Corporation, Tokyo, Japan).

Western blot analysis. The western blotting procedures were conducted as described in previous studies $(9,10)$. The cells treated with Tan-IIA were lysed in ice-cold radioimmunoprecipitation assay buffer (Merck Millipore) containing a protease inhibitor cocktail (Gibco; Thermo Fisher Scientific, Inc.). The lysate was agitated for $30 \mathrm{~min}$ at $4^{\circ} \mathrm{C}$ and centrifuged at $12,281 \mathrm{x} \mathrm{g}$ for $10 \mathrm{~min}$. The protein concentration was measured using the Pierce ${ }^{\mathrm{TM}}$ BCA Protein Assay kit (Thermo Fisher Scientific, Inc.). Equal quantities of proteins $(10 \mu \mathrm{g})$ were subjected to electrophoresis using $12 \%$ sodium dodecyl sulfate-polyacrylamide gels (Bio-Rad Laboratories, Inc., Hercules, CA, USA; stacking gel: 70 V, 400 mA, 30 min; separating gel: $100 \mathrm{~V}, 400 \mathrm{~mA}, 90 \mathrm{~min})$. To verify equal protein loading and transfer, the proteins were transferred to PVDF membranes, which were blocked for $1 \mathrm{~h}$ at $4^{\circ} \mathrm{C}$ using blocking buffer [5\% dried, skimmed milk in solution containing $50 \mathrm{mM}$ Tris- $\mathrm{HCl}$ ( $\mathrm{pH} \mathrm{8.0),} 2 \mathrm{mM} \mathrm{CaCl}_{2}, 80 \mathrm{mM}$ sodium chloride, $0.05 \%$ Tween-20 and $0.02 \%$ sodium azide (Merck Millipore)]. The membranes were subsequently incubated for $2 \mathrm{~h}$ at room temperature with the following primary antibodies: Rabbit polyclonal anti-NF- $\mathrm{kB}-\mathrm{p} 65$, monoclonal rabbit anti-COX-2, monoclonal rabbit anti-MMP-2, polyclonal goat anti-MMP-7, polyclonal rabbit anti-MMP-9 (all diluted to 1:1,000), followed by incubation at room temperature for $1 \mathrm{~h}$ with anti-rabbit (cat. no. sc:2004) or anti-mouse (cat. no. sc:2005) IgG-horseradish peroxidase-conjugated secondary antibodies (1:5,000; Santa Cruz Biotechnology Inc., Santa Cruz, CA, USA). The membranes were washed three times for $10 \mathrm{~min}$ with $1 \mathrm{X}$ phosphate-buffered saline with $0.05 \%$ (v/v) Tween 20 . 
A

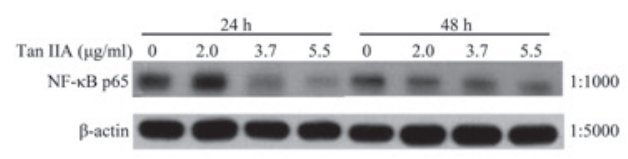

B

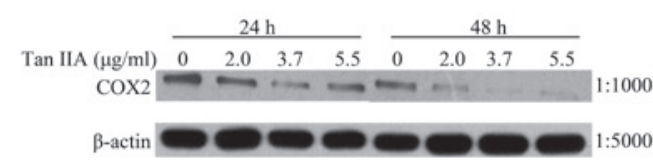

C

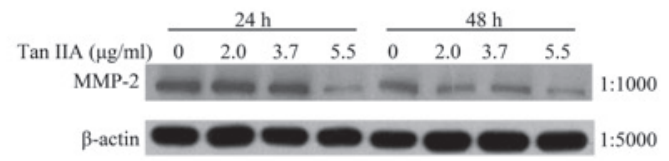

D

E
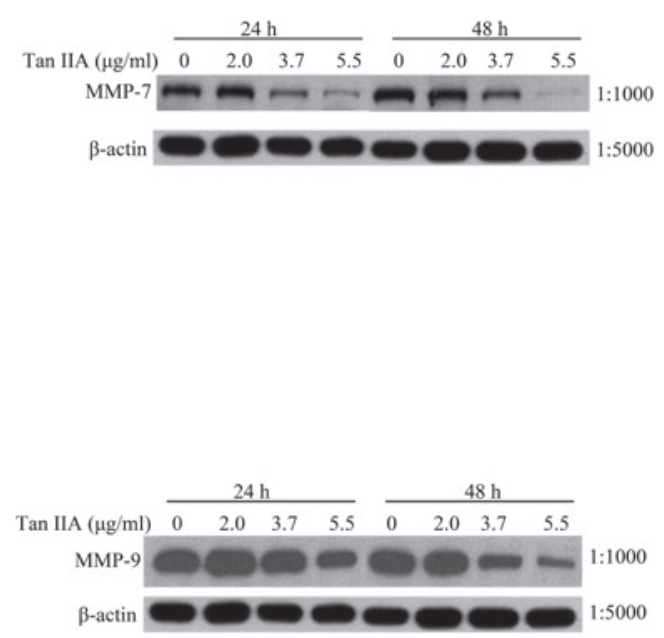
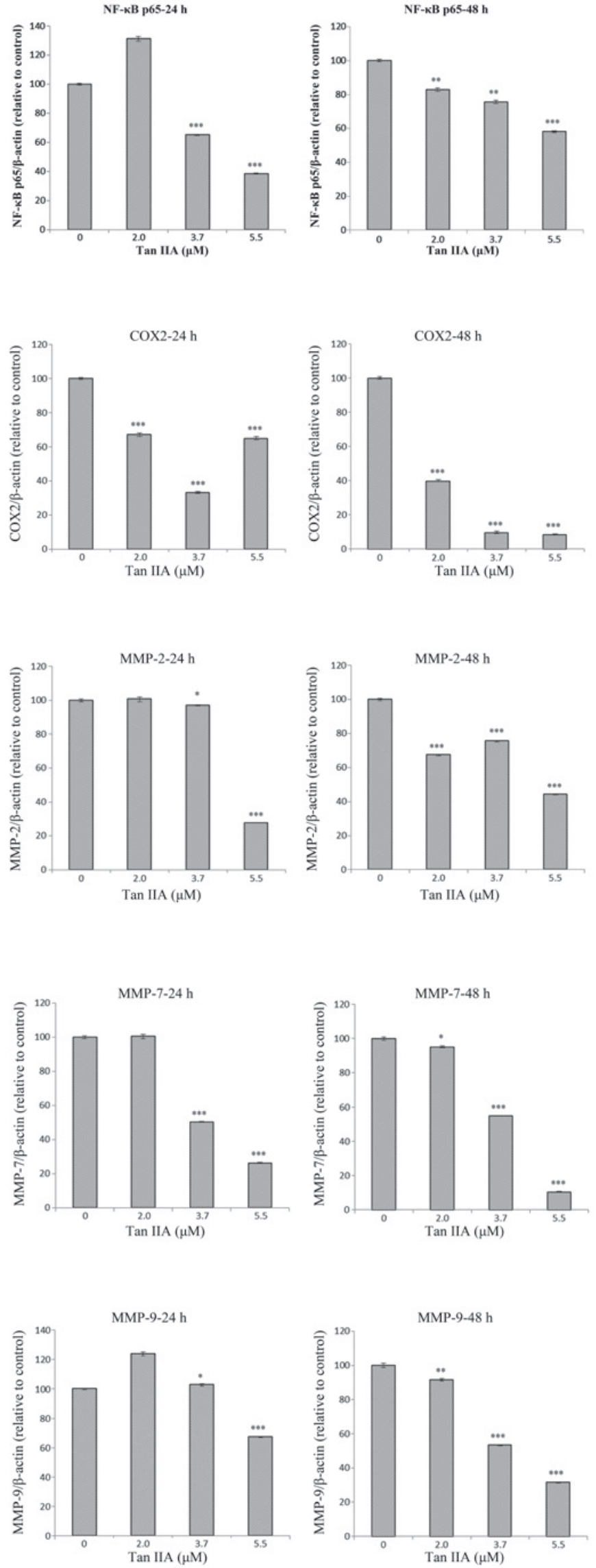

Figure 2. Protein expression levels of NF- $\kappa$ B-p65, COX-2, MMP-2, -7, and -9 and $\beta$-actin in AGS cells. The AGS cells were treated with various concentrations of Tan-IIA $(0,2.0,3.7$ and $5.5 \mu \mathrm{g} / \mathrm{ml})$ for 24 or $48 \mathrm{~h}$ and the protein expression levels were evaluated by western blot analysis. The results indicate that Tan-IIA significantly decreases the protein expression levels of (A) NF- $\mathrm{B}-\mathrm{p} 65$, (B) COX-2, (C) MMP-2, (D) MMP-7 and (E) MMP-9 in a dose-dependent manner. Tan-IIA, tanshinone IIA; NF- $\kappa \mathrm{B}-\mathrm{p} 65$, nuclear factor $\kappa \mathrm{B}-\mathrm{p} 65$; COX-2, cyclooxygenase-2; MMP, matrix metalloproteinase. ${ }^{*} \mathrm{P}<0.05$, ${ }^{* *} \mathrm{P}<0.01$ and ${ }^{* * *} \mathrm{P}<0.001$, compared with the control. 
A
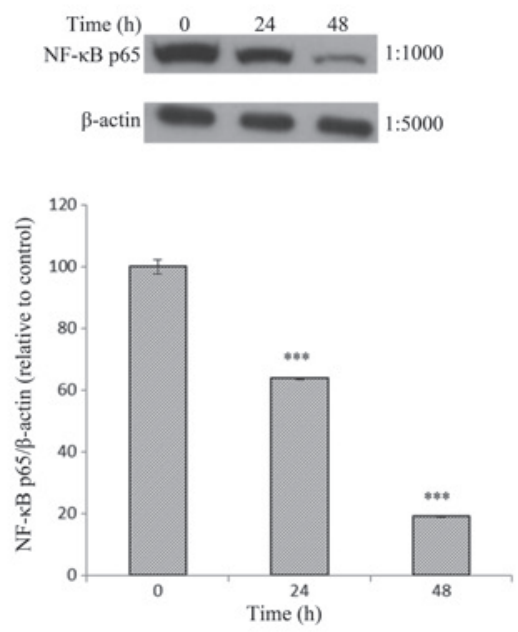

C
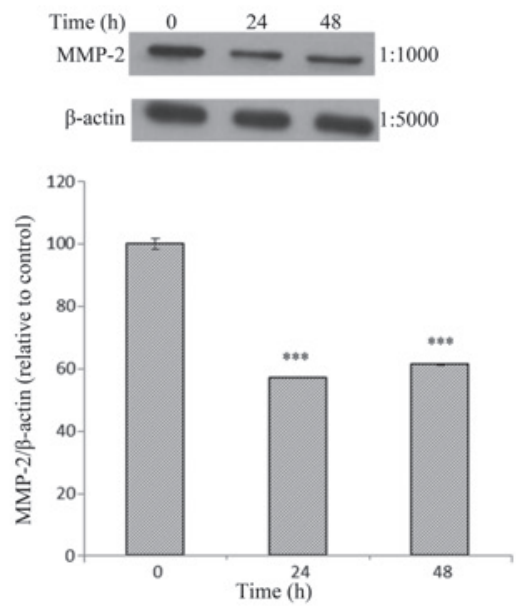
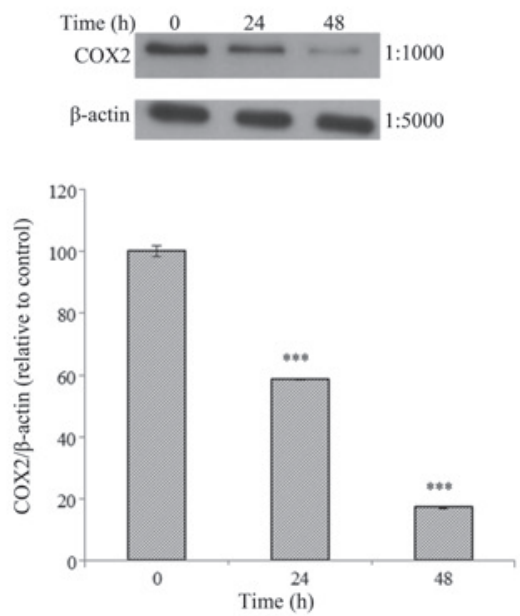

D

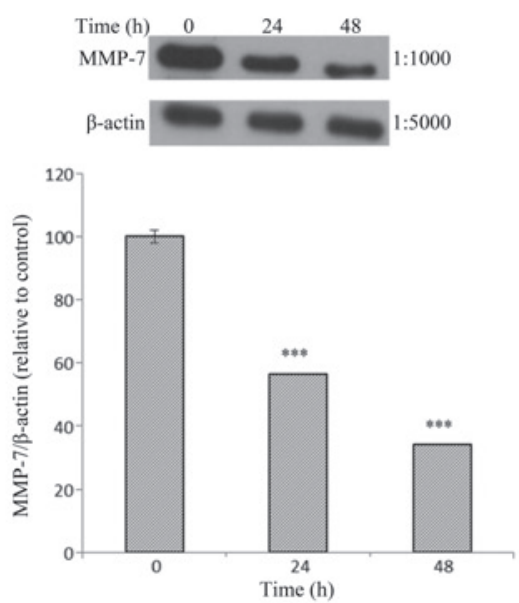

$\mathbf{E}$
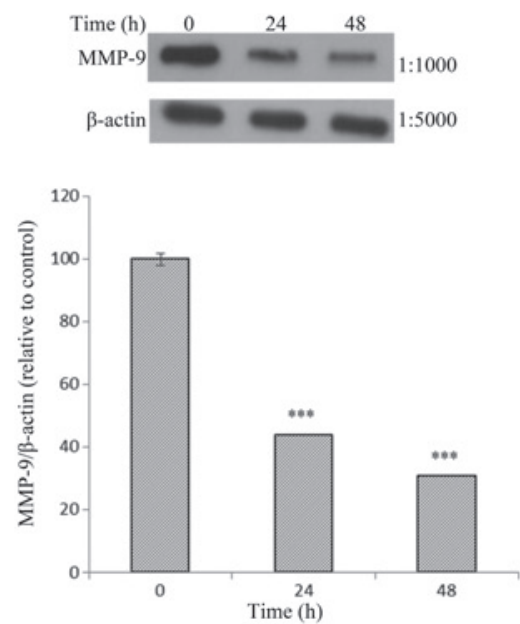

Figure 3. Protein expression levels of NF-кB-p65, COX-2, MMP-2, -7, and -9 and $\beta$-actin in AGS cells. The AGS cells were treated with Tan-IIA (3.7 $\mu$ g/ml) for different durations $(0,24$ and $48 \mathrm{~h})$ and the protein expression levels were evaluated by western blot analysis. The results demonstrate that Tan-IIA significantly decreases the protein expression levels of (A) NF-кB-p65, (B) COX-2, (C) MMP-2, (D) MMP-7 and (E) MMP-9 in a time-dependent manner

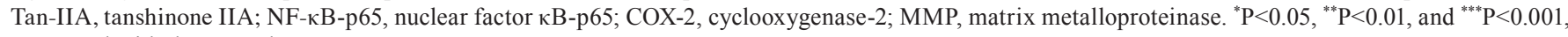
compared with the control.

The protein bands were visualized on X-ray film using an enhanced chemiluminescence detection system (PerkinElmer, Inc., Waltham, MA, USA).
Statistical analysis. All data presented are from a minimum of three independent experiments. Values are presented as the mean \pm standard deviation. Student's t-test was used to analyze 
statistical significance and $\mathrm{P}<0.05$ was considered to indicate a statistically significant difference.

\section{Results}

Wound-healing assays. The wound-healing assay is one of the methods used to investigate cell migration in vitro. A wound was created in the cell monolayer using a plastic pipette tip and AGS cells were treated with various concentration of Tan-IIA $(0,2.0,3.7$ and $5.5 \mu \mathrm{g} / \mathrm{ml})$ for 24 or $48 \mathrm{~h}$. Images were captured to compare the cell migration for wound closure. The results indicate that Tan-IIA inhibits the migration of AGS cells in a time- and dose-dependent manner (Fig. 1).

Effects of Tan-IIA concentration on the protein expression levels of $N F-\kappa B-p 65, C O X-2, M M P-2,-7$, and -9 and $\beta$-actin in AGS cells. The AGS cells were treated with various concentrations of Tan-IIA $(0,2.0,3.7$ and $5.5 \mu \mathrm{g} / \mathrm{ml})$ for 24 or $48 \mathrm{~h}$ and the protein expression levels of NF-кB-p65, COX-2, MMP-2, -7, and -9 and $\beta$-actin were evaluated by western blot analysis. The results demonstrate that Tan-IIA significantly decreases the protein expression levels of NF- $\mathrm{B}-\mathrm{p} 65$ (Fig. 2A), COX-2 (Fig. 2B), and MMP-2 (Fig. 2C), -7 (Fig. 2D) and -9 (Fig. 2E) in a dose-dependent manner.

Effects of Tan-IIA treatment duration on the protein expression of NF- $\kappa B-p 65, C O X-2, M M P-2,-7$, and -9 and $\beta$-actin in AGS cells. The AGS cells were treated with Tan-IIA $(3.7 \mu \mathrm{g} / \mathrm{ml})$ for different durations $(0,24$ and $48 \mathrm{~h})$ and the protein expression levels were evaluated by western blot analysis. The results demonstrate that Tan-IIA significantly decreases the protein expression levels of NF- $\mathrm{B}-\mathrm{p} 65, \mathrm{COX}-2$, and MMP-2, -7 and -9 in a time-dependent manner (Fig. 3).

\section{Discussion}

Cell migration in vitro is commonly evaluated using a wound-healing assay. A wound is created in the cell monolayer using a plastic pipette tip. Images are captured upon initiation of the assay and at regular time-points during cell migration (that is taking place to close the wound), and these images are compared to measure the cell migration. This method aims to mimic cell migration during wound healing in vivo (16). The present study indicates that Tan-IIA inhibits the migration ability of AGS human gastric cancer cells in a time- and dose-dependent manner in vitro. This finding is consistent with previous studies, which demonstrated that Tan-IIA markedly decreased migratory and invasive abilities in SGC7901 gastric cancer cells (15). It is well known that MMP-2 overexpression is significantly associated with poor overall survival (OS) of gastric cancer patients, and has a negative impact on OS in Asian and European countries (17). A previous meta-analysis indicated that MMP-2 overexpression may be a predictive factor for poor prognosis in gastric cancer (17). de la Peña et al (18) demonstrated that MMP-2 expression may present as a potential molecular marker for advanced human gastric cancer. An additional meta-analysis indicated that abnormal MMP-2 expression may be markedly associated with poor prognosis in gastric cancer patients (19). Yang et al (20) demonstrated that the MMP-7-181 A>G polymorphism may contribute to gastric cancer susceptibility. Furthermore, Huang et al (21) demonstrated that interleukin (IL)-1 $\beta$-induced p38 activation significantly increased the messenger (m)RNA and protein expression, and activity of MMP-2 and -9. This indicated the IL-1 $\beta / \mathrm{p} 38 /$ activator protein 1 (c-Fos)/MMP-2 and -9 signaling pathway may be important in metastasis in MKN-45 and AGS cells. In our previous study, AGS cells were treated with Tan-IIA and it was demonstrated that treatment increased p-p38 expression levels in a time- and dose-dependent manner (10). In the present study, the results indicate that Tan-IIA decreases MMP-2, -7 and -9 expression levels in a time- and dose-dependent manner. The results indicate that Tan-IIA may inhibit the metastasis of AGS cells by decreasing MMP-2, -7 and -9 expression levels.

Carcinogen exposure and chronic inflammation are significant underlying conditions resulting in tumor development. It is well documented that $\mathrm{NF}-\kappa \mathrm{B}$ is essential in promotion of inflammation-associated cancer, and is therefore being considered as a potential target for cancer prevention in chronic inflammatory diseases (11). MGC803 gastric cancer cells treated with chloroquine inhibited the mRNA expression levels of COX-2, MMP-2 and -7 and $\mathrm{NF}-\kappa \mathrm{B}-\mathrm{p} 65$. This prevented the migration of MGC803 cells in a dose-dependent manner. Furthermore, the results indicated that the Toll-like receptor $9 / \mathrm{NF}-\kappa \mathrm{B}$ signaling pathway was involved in gastric cancer cell migration (22). The results from the current study indicate that Tan-IIA decreases NF- $\mathrm{B}$-p 65 and COX-2 expression levels in a time- and dose-dependent manner. In addition, the results from the wound-healing assay indicate that Tan-IIA inhibits the migration of AGS cells for wound closure in a time- and dose-dependent manner. These results suggest that Tan-IIA inhibits the migration ability of AGS cells by decreasing the protein expression of NF- $\mathrm{KB}-\mathrm{p} 65, \mathrm{COX}-2$, and MMP-2, -7 and -9 .

In conclusion, to the best of our knowledge, this is the first study to report that Tan-IIA inhibits the migration ability of AGS cells via decreasing the protein expression of NF- $\kappa \mathrm{B}-\mathrm{p} 65$, COX-2 and MMPs. H owever, these findings warrant further investigation in the future.

\section{Acknowledgements}

The present study was supported by a grant from the Research Section of the Changhua Christian Hospital (Changhua, Taiwan; grant no. 102-CCH-IRP-066).

\section{References}

1. Che AJ, Zhang JY, Li CH, Chen XF, Hu ZD and Chen XG: Separation and determination of active components in Radix Salviae miltiorrhizae and its medicinal preparations by nonaqueous capillary electrophoresis. J Sep Sci 27: 569-575, 2004.

2. Zhou L, Zuo Z and Chow MS: Danshen: An overview of its chemistry, pharmacology, pharmacokinetics, and clinical use. J Clin Pharmacol 45: 1345-1359, 2005.

3. Chiu TL and Su CC: Tanshinone IIA induces apoptosis in human lung cancer A549 cells through the induction of reactive oxygen species and decreasing the mitochondrial membrane potential. Int J Mol Med 25: 231-236, 2010.

4. Su CC and Lin YH: Tanshinone IIA inhibits human breast cancer cells through increased Bax to Bcl-xL ratios. Int J Mol Med 22: $357-361,2008$. 
5. Yan MY, Chien SY, Kuo SJ, Chen DR and Su CC: Tanshinone IIA inhibits BT-20 human breast cancer cell proliferation through increasing caspase 12 , GADD153 and phospho-p38 protein expression. Int J Mol Med 29: 855-863, 2012.

6. Cheng CY and Su CC: Tanshinone IIA inhibits Hep-J5 cells by increasing calreticulin, caspase 12 and GADD153 protein expression. Int J Mol Med $26: 379-385,2010$.

7. Huang CY, Chiu TL, Kuo SJ, Chien SY, Chen DR and Su CC: Tanshinone IIA inhibits the growth of pancreatic cancer BxPC 3 cells by decreasing protein expression of TCTP, MCL 1 and Bcl-xL. Mol Med Rep 7: 1045-1049, 2013.

8. Su CC, Chen GW, Kang JC and Chan MH: Growth inhibition and apoptosis induction by tanshinone IIA in human colon adenocarcinoma cells. Planta Med 74: 1357-1362, 2008.

9. Su CC: Tanshinone IIA inhibits human gastric carcinoma AGS cell growth by decreasing BiP, TCTP, Mcl 1 and Bcl-xL and increasing Bax and CHOP protein expression. Int J Mol Med. 34: $1661-1668,2014$

10. Su CC: Tanshinone IIA inhibits gastric carcinoma AGS cells through increasing p-p38, p-JNK and p53 but reducing p-ERK, CDC2 and cyclin B1 expression. Anticancer Res 34: 7097-7110, 2014.

11. Pikarsky E, Porat RM, Stein I, Abramovitch R, Amit S, Kasem S, Gutkovich-Pyest E, Urieli-Shoval S, Galun E and Ben-Neriah Y: NF-kappaB functions as a tumour promoter in inflammation-associated cancer. Nature 431:461-466, 2004.

12. Wu CY, Wu MS, Chen YJ, Chen CJ, Chen HP, Shun CT, Chen GH, Huang SP and Lin JT: Clinicopathological significance of MMP-2 and TIMP-2 genotypes in gastric cancer. Eur J Cancer 43: 799-808, 2007.

13. Egeblad M and Werb Z: New functions for the matrix metalloproteinases in cancer progression. Nat Rev Cancer 2: 161-174, 2002.
14. Elnemr A, Yonemura Y, Bandou E, Kinoshita K, Kawamura T, Takahashi S, Tochiori S, Endou Y and Sasaki T: Expression of collagenase-3 (matrix metalloproteinase-13) in human gastric cancer. Gastric Cancer 6: 30-38, 2003.

15. Xu M, Cao FL, Li NY, Liu YQ, Li YP and Lv CL: Tanshinone IIA reverses the malignant phenotype of SGC7901 gastric cancer cells. Asian Pac J Cancer Prev 14: 173-177, 2013.

16. Rodriguez LG, Wu X and Guan JL: Wound-healing assay. Methods Mol Biol 294: 23-29, 2005.

17. Shen W, Xi H, Wei B and Chen L: The prognostic role of matrix metalloproteinase 2 in gastric cancer: A systematic review with meta-analysis. J Cancer Res Clin Oncol 40: 1003-1009, 2014.

18. de la Peña S, Sampieri CL, Ochoa-Lara M, León-Córdoba K and Remes-Troche JM: Expression of the matrix metalloproteases $2,14,24$, and 25 and tissue inhibitor 3 as potential molecular markers in advanced human gastric cancer. Dis Markers 2014: 285906, 2014.

19. Wang HL, Zhou PY, Zhang Y and Liu P: Relationships between abnormal MMP2 expression and prognosis in gastric cancer: A meta-analysis of cohort studies. Cancer Biother Radiopharm 29: 166-172, 2014.

20. Yang TF, Guo L and Wang Q: Meta-analysis of associations between four polymorphisms in the matrix metalloproteinases gene and gastric cancer risk. Asian Pac J Cancer Prev 15: 1263-1267, 2014.

21. Huang Q, Lan F, Wang X, Yu Y, Ouyang X, Zheng F, Han J, Lin Y, Xie Y, Xie F, et al: IL-1 $\beta$-induced activation of p38 promotes metastasis in gastric adenocarcinoma via upregulation of AP-1/c-fos, MMP2 and MMP9. Mol Cancer 31: 13-18, 2014.

22. Zhang Y, Li Y, Li Y, Li R, Ma Y, Wang H and Wang Y: Chloroquine inhibits MGC803 gastric cancer cell migration via the Toll-like receptor 9/nuclear factor kappa B signaling pathway. Mol Med Rep 11: 1366-1371, 2015. 